Computational and Mathematical Methods in Medicine

Vol. 9, Nos. 3-4, September-December 2008, 339-349

Taylor \& Francis

Taylor \& Francis Group

\title{
RNA packing specificity and folding during assembly of the bacteriophage MS2
}

Ottar Rolfsson, Katerina Toropova, Victoria Morton, Simona Francese ${ }^{1}$, Gabriella Basnak, Gary S. Thompson, Stephen W. Homans, Alison E. Ashcroft, Nicola J. Stonehouse, Neil

A. Ranson and Peter G. Stockley*

Astbury Centre for Structural Molecular Biology, University of Leeds, Leeds, UK

(Received 18 February 2008; final version received 1 March 2008)

\begin{abstract}
Using a combination of biochemistry, mass spectrometry, NMR spectroscopy and cryoelectron microscopy (cryo-EM), we have been able to show that quasi-equivalent conformer switching in the coat protein (CP) of an RNA bacteriophage (MS2) is controlled by a sequence-specific RNA-protein interaction. The RNA component of this complex is an RNA stem-loop encompassing just $19 \mathrm{nts}$ from the phage genomic RNA, which is $3569 \mathrm{nts}$ in length. This binding results in the conversion of a $\mathrm{CP}$ dimer from a symmetrical conformation to an asymmetric one. Only when both symmetrical and asymmetrical dimers are present in solution is assembly of the $T=3$ phage capsid efficient. This implies that the conformers, we have characterized by NMR correspond to the two distinct quasi-equivalent conformers seen in the $3 \mathrm{D}$ structure of the virion. An icosahedrally-averaged single particle cryo-EM reconstruction of the wild-type phage (to $\sim 9 \AA$ resolution) has revealed icosahedrally ordered density encompassing up to $90 \%$ of the single-stranded RNA genome. The RNA is seen with a novel arrangement of two concentric shells, with connections between them along the 5 -fold symmetry axes. RNA in the outer shell interacts with each of the $90 \mathrm{CP}$ dimers in the $T=3$ capsid and although the density is icosahedrally averaged, there appears to be a different average contact at the different quasi-equivalent protein dimers: precisely the result that would be expected if protein conformer switching is RNA-mediated throughout the assembly pathway. This unprecedented RNA structure provides new constraints for models of viral assembly and we describe experiments aimed at probing these. Together, these results suggest that viral genomic RNA folding is an important factor in efficient assembly, and further suggest that RNAs that could sequester viral CPs but not fold appropriately could act as potent inhibitors of viral assembly.
\end{abstract}

Keywords: RNA-protein interactions; MS2; ssRNA virus; genomic RNA structure and folding; cryo-electron microscopy; viral assembly

\section{Introduction}

The ssRNA viruses are one of the largest groups of viruses, and include human immunodeficiency virus, hepatitis $\mathrm{C}$ virus, Norwalk viruses and the Picornaviridae such as rhinovirus, poliovirus, hepatitis A and foot and mouth disease virus, as well as plant viruses such as tomato bushy stunt virus [25]. These viral pathogens infect human, animal and plant hosts and for many of these novel routes towards anti-viral therapy are urgently required. All these viruses face the common challenge of packaging their nucleic acids into protective shells composed of a defined number of one, or very few, type(s) of coat protein (CP) subunits, reflecting constraints of genetic

\footnotetext{
*Corresponding author. Email: stockley@ bmb.leeds.ac.uk
} 
economy and quasi-equivalence [6,8]. Many ssRNA viruses have evolved lifecycles during which capsid assembly is triggered by sequence/structure-specific interaction between CPs and their RNA genomes [26,38]. A major unresolved problem in structural virology, however, has been to understand the detailed molecular mechanism(s) that gives rise to the highly efficient assembly of capsids of the correct size and symmetry in such systems. This is especially true for viruses whose capsids exhibit quasi-equivalent symmetry in their CP lattices [6]. Various proposals for formation of protein shells based on the initial formation of 3-fold [26], or 5-fold [23] assembly initiation complexes have been made previously. Capsid assembly is, however, spontaneous and usually very rapid, with the result that it has been technically difficult to isolate and characterize intermediates on the pathway to the final products beyond these initiation complexes' [22,41]. Understanding these processes in mechanistic detail may well lead to novel routes for anti-viral therapy.

The RNA bacteriophage MS2 is an ideal model for investigating such phenomena owing to the extensive biochemical and structural information that is available [12,14,27,34,35,39]. We have therefore undertaken detailed mechanistic and structural studies of this phage in order to address the specific questions of how the genomic RNA is encapsidated specifically, how the formation of the $T=3$ quasi-equivalent shell is controlled during the assembly reaction, and what role(s), if any, the ssRNA genome plays during these processes. MS2 is a member of the Leviviridae family of viruses that infect male Escherichia coli cells via an initial attachment to the bacterial F-pilus [21,27]. It has a single-stranded, positive-sense RNA genome of 3569 nucleotides that encodes just four gene products: $\mathrm{CP}$, replicase, lysis protein and maturation protein (Figure 1). CP is the most highly expressed of the gene products and 180 copies assemble to form an icosahedral protein shell that encapsidates the genome in the mature virion. A single copy of the maturation protein is also incorporated into the virion and functions during infection by binding the F-pilus of target cells. A complex between the maturation protein and the genomic RNA is the only viral component to enter host cells during infection [40].

The crystal structure of the wild-type MS2 bacteriophage has been determined to $2.8 \AA$ resolution [9,34]. The $\mathrm{CP}$ fold was unique amongst known icosahedral viruses at that time, although it has since been shown to be shared by the other Leviviridae CPs [32]. The main chain folds into a five-stranded anti-parallel $\beta$-sheet with two anti-parallel $\beta$-strands folding over it at the $\mathrm{N}$-terminus and with a kinked $\alpha$-helix at the $\mathrm{C}$-terminus (Figure 2). The $\mathrm{C}$-terminal $\alpha$-helices of two $\mathrm{CP}$ monomers interdigitate to form non-covalent dimers $\left(\mathrm{CP}_{2}\right)$ in the capsid and these can be isolated as dimers in solution by acid dissociation [31]. In the $T=3$ capsid, the $\mathrm{CP}$ is found in three distinct conformations, termed A, B and C, consistent with the quasi-equivalent symmetry required to construct a $T=3$ structure. The main site of variation between conformers is in the loop between the $\mathrm{F}$ and $\mathrm{G}, \beta$-strands (FG-loop), which is extended in A and $\mathrm{C}$ conformers, but bent back toward the main body of the subunit in the $\mathrm{B}$ conformer (Figure 2). The capsid thus contains two types of dimer; an asymmetric $\mathrm{A} / \mathrm{B}$ dimer, and a symmetric $\mathrm{C} / \mathrm{C}$ dimer. The positioning of these different building blocks within the protein shell controls the size and symmetry of the viral particle. Unusually, the capsid has large ( $\sim 14 \AA$ dia.) pores at the 5-fold symmetry axes and it is not known whether these play a role in the viral life cycle, although, mutation of a conserved proline residue (Pro78) lining the pore at 5-fold axes results in production of assembled $T=3$ capsids but fails to produce phage from an infectious recombinant clone [11].

Capsid reassembly can be triggered in vitro by a sequence-specific RNA-protein interaction between acid-disassembled CP subunits, which form dimers at more neutral pHs and an RNA stem-loop (TR) of just $19 \mathrm{nt}$ derived from the genomic sequence that encompasses the start codon of the viral replicase ([2,3]; Figure 2). RNA-protein binding thus achieves two functions via a single molecular recognition event: translational repression of the replicase and creation of an assembly competent complex on viral RNA. 

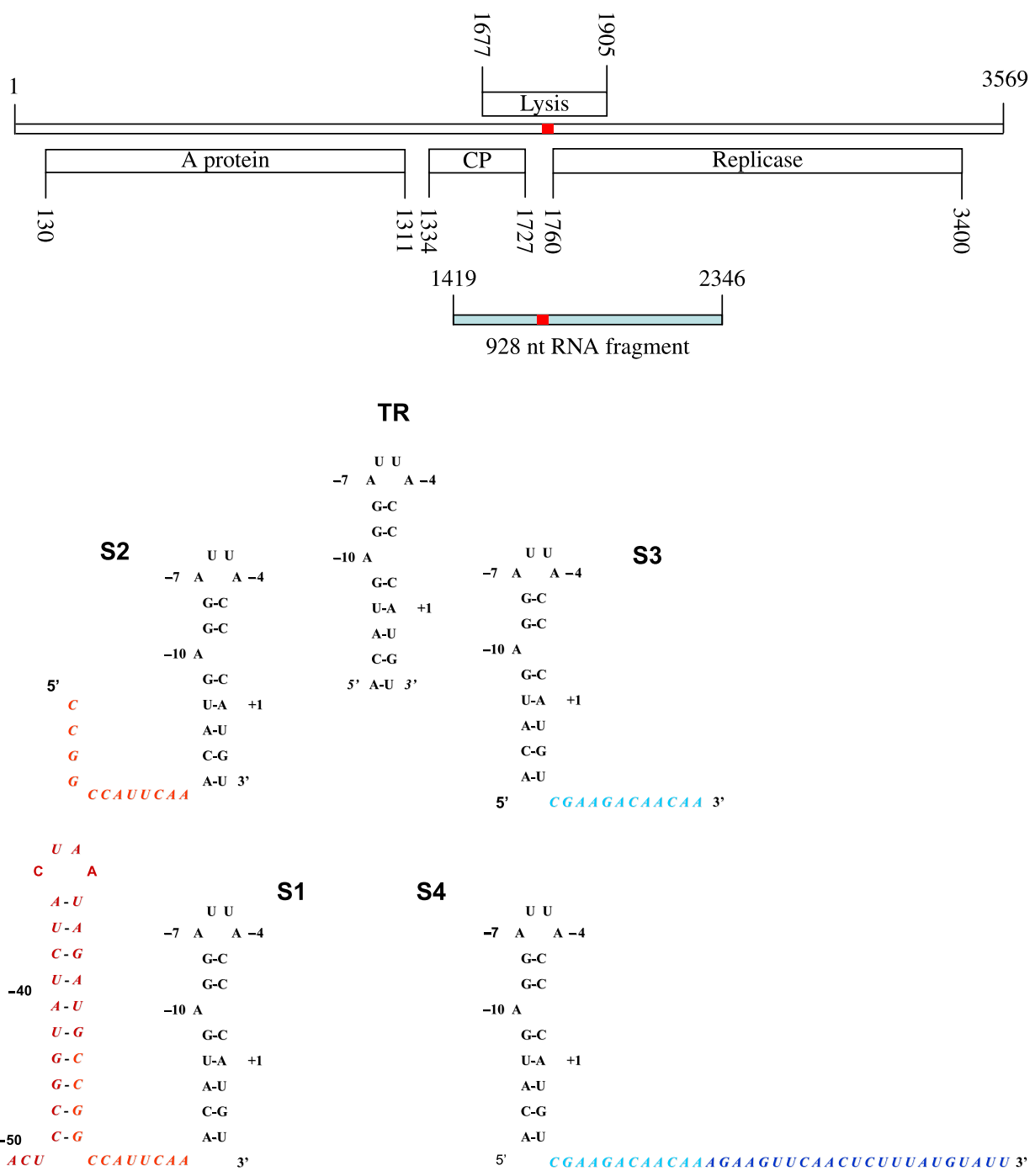

Figure 1. Genomic map of bacteriophage MS2 and sequences of the RNAs used in reassembly experiments. The upper panel shows a diagrammatic representation of the single-stranded $3569 \mathrm{nt}$ long MS2 genome and the positions of the genes it encodes. The red bar shows the location of the TR assembly initiation stem-loop. The sub-genomic fragment is shown highlighted in light blue. The lower panel shows the sequences and putative secondary structures of the short RNA fragments used for reassembly experiments. Fragments S1-S4 represent 12 and 35 nt extensions at the $5^{\prime}$ (increasing red) or $3^{\prime}$ (increasing blue) ends of the TR stem-loop, respectively. The TR fragment is numbered with respect to the start codon of the replicase cistron $\left(\mathrm{A}_{=1} \mathrm{UG}\right)$.

\section{Results and discussion}

\section{Specific encapsidation of the short genomic RNA}

Results from co-infections by two closely related RNA phages, MS2 and Q $\beta$, in the same bacterial cell suggest that there is sequence selective encapsidation by the cognate $\mathrm{CP}$ subunits of their respective genomic RNAs [16]. The molecular basis of this selectivity has been established by the pioneering in vitro experiments of Uhlenbeck and colleagues, who showed 

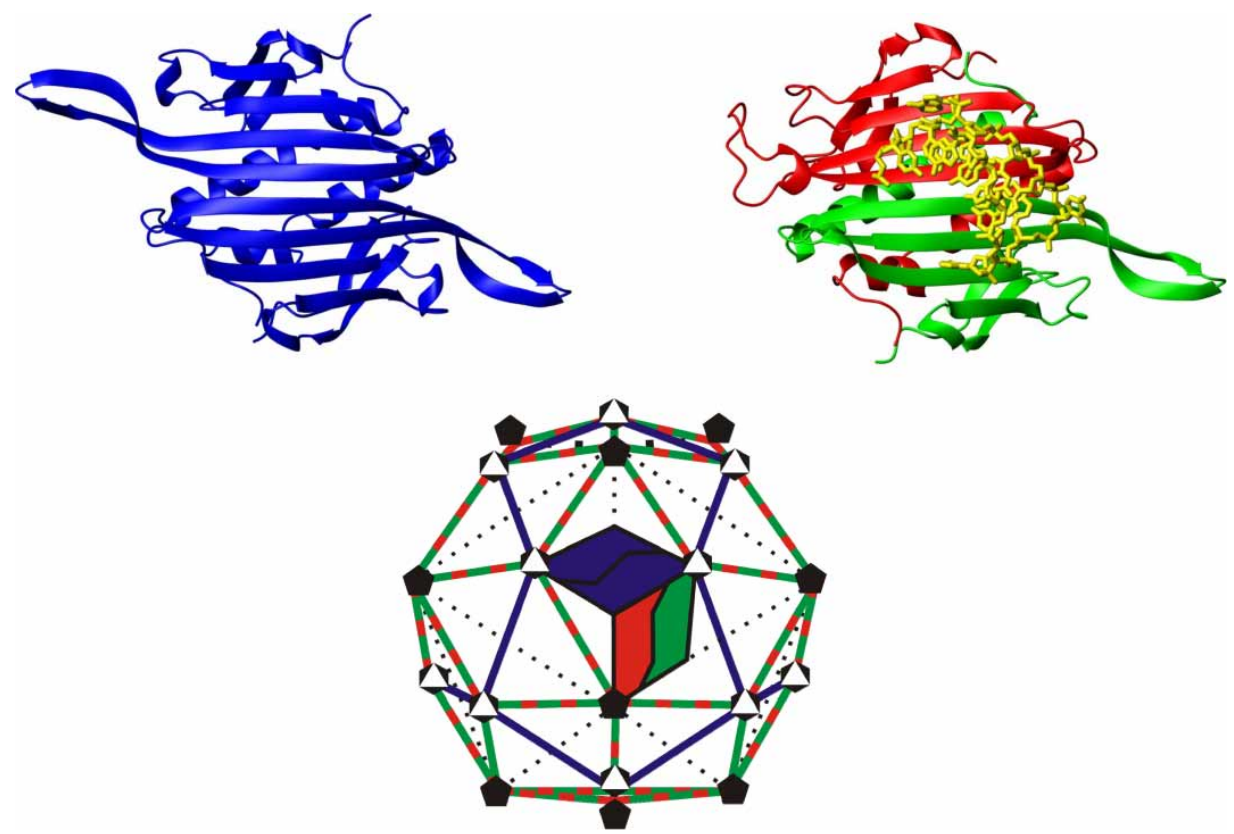

Figure 2. Structures of the MS2 quasi-equivalent coat protein dimers and their relationship within a $T=3$ shell. The $\mathrm{C} / \mathrm{C}$ (red/red) and $\mathrm{A} / \mathrm{B}$ (blue/green) quasi-equivalent dimers are shown as ribbon representations viewed along the 2 -fold axes from the inside of the phage particle. The TR RNA (yellow stick model) is shown bound to the A/B dimer. Structures are taken from pdb1ZDH. The relationship of A/B and C/C dimers to the final $T=3$ capsid is shown below. Adapted from Ref. [29]. Available in colour online.

that sequence-specific interaction between R17 (an MS2 homologue) and its genomic RNA was controlled by a single $19 \mathrm{nt}$ stem-loop within the $3569 \mathrm{nt}$ genomic RNA [4,5]. We have recently extended these studies using X-ray crystallography to provide the first atomic level mechanism for packaging discrimination [12]. The in vitro affinity of the MS2 stem-loop for its CP is very similar to that for the intact genomic RNA. This recognition sequence encompasses the $5^{\prime}$ start codon of the phage replicase gene, and therefore also provides a molecular explanation for the translational repression of the replicase cistron by CP expression [17]. The 'appearance' of the $19 \mathrm{nt}$ translational operator stem-loop (TR) is controlled by phage gene expression. Initially, following phage RNA entry into the bacterium, the TR sequence is sequestered in a long-range secondary structure, base pairing with a portion of the CP gene - the 'Min Jou' sequence [13]. $\mathrm{CP}$ gene translation is required to free the TR sequence and initiate folding into the stem-loop operator. Beckett and Ulhenbeck showed that in isolation this TR fragment triggers reassembly of $T=3$ shells by the CP more efficiently than polyA [2,3].

These observations raise a number of important questions. How does the TR stem-loop fold into the operator conformation? How is the TR sequence recognized specifically by the $\mathrm{CP}$ dimer? How does the $\mathrm{CP}$ dimer $\left(\mathrm{CP}_{2}\right)$ : TR complex lead to assembly of a shell with the correct size and symmetry? Single-molecule fluorescence data, that will be described in detail elsewhere (Gell et al. in prep), suggest that TR folds as a two-state system, consistent with its role as a genetic switch. Previous stopped-flow fluorescence measurements suggest that isolated $19 \mathrm{nt}$ stem-loops bind to $\mathrm{CP}$ dimers in a diffusion-controlled reaction, although, these data showed some evidence that protein conformational changes were occurring during the binding [14].

Expression of a recombinant MS2 CP gene in E. coli leads to formation of $T=3$ shells containing small amounts of cellular RNA [18]. These are easily purified and crystallize 
isomorphously with the wild-type phage, even though they lack the genomic RNA and the single copy of the maturation protein. Soaking RNA oligonucleotides encompassing the TR sequence [35], sequence or chemical variants [10,36], or even aptamer consensus sequences [7,24], into such crystals allows the RNA to access the inner surface of the protein shell, presumably via the pores through the capsid, where they bind to every $\mathrm{CP}$ dimer. This technique has allowed the 3D structures of a large number of different RNA-protein complexes to be determined by X-ray diffraction difference maps. TR binds to A/B quasi-equivalent dimers in a unique orientation, allowing the interaction to be resolved at atomic resolution (Figure 2). It is clear from such experiments that the TR binds across the inner face of the $\mathrm{CP}_{2}$, a sheet comprising ten $\beta$-strands. The RNA in this site is at least $12 \AA$ away from the nearest amino acid residues of the loops whose conformations define the quasi-symmetry of the capsid. Building a $T=3$ capsid requires these loop conformations to be set in a local symmetry context and their separation from TR binding site does not immediately reveal the mechanism underlying this switch. The conformation of the TR bound to protein differs from that seen in solution because the $-10 \mathrm{~A}$ residue (see Figure 1 legend for numbering scheme) becomes extruded from the stem, which takes up an A duplex structure. The - $10 \mathrm{~A}$ base and the loop residue $-4 \mathrm{~A}$ bind into identical pockets, but in different orientations, on the surface of each protein monomer. Thus, the bulk conformation of the CP dimer is unchanged by binding TR RNA, consistent with the very rapid on rate observed in the stopped-flow experiments [14]. These studies therefore did not clarify how quasi-equivalent conformer switching is achieved during assembly.

\section{The mechanism of $\mathrm{T}=3$ shell formation}

The major insight into the molecular mechanism controlling $T=3$ shell formation was made by studying TR-mediated reassembly of CP dimers by non-covalent mass spectrometry [29]. Reaction mixtures containing equimolar amounts of $\mathrm{CP}_{2}$ and TR rapidly formed the 'initiating' RNA-protein complex but these were only very slowly converted into species with higher stoichiometries. Addition of a further aliquot of $\mathrm{CP}_{2}$ lacking $\mathrm{RNA}$, however, resulted in rapid formation of higher order species including broad peaks in the mass spectrum that correspond to the peaks observed when the recombinant $T=3$ shell is analysed. It appears that both RNAbound and RNA-free forms of the $\mathrm{CP}_{2}$ are required for efficient and rapid capsid assembly.

Since there are two types of $\mathrm{CP}_{2}$ dimer conformer in the final capsid this result is suggestive of RNA-binding resulting in conformational change in the protein dimer. This possibility was investigated using NMR spectroscopy to determine the chemical shift values of the CP subunits with and without bound TR using a protein mutant, W82R that does not assemble beyond dimer [20]. The data showed very clearly that in the absence of RNA the chemical shifts of the residues in the FG-loop are the same in both subunits, whereas they become distinct when the TR stem loop is bound. The RNA binding site is sufficiently far from the FG-loops that these effects are not due to direct alteration in the local chemical environment. In other words, the NMR data show unambiguously that TR acts allosterically to promote formation of an asymmetric (presumably A/B-like) $\mathrm{CP}_{2}$ from an RNA-free species that is symmetric. Analysis of the intermediate species in the mass spectra of the reassembly reaction (Figure 3) revealed the presence of complexes with the following stoichiometries: $\mathrm{CP}_{2}$ :TR of mass $33.5 \mathrm{kDa}$ (Figure 3; component A), [3( $\left.\left.\mathrm{CP}_{2}: \mathrm{TR}\right)+3 \mathrm{CP}_{2}\right]$ of mass $182.7 \mathrm{kDa}$ (Figure 3; component $\mathrm{B}$ ), $\left[3\left(\mathrm{CP}_{2}: \mathrm{TR}\right)+2 \mathrm{CP}_{2}+\mathrm{CP}\right]$ of mass $169.2 \mathrm{kDa}$ (Figure 3 ; component $\mathrm{C}$ ) and $\left[5\left(\mathrm{CP}_{2}: \mathrm{TR}\right)+5 \mathrm{CP}_{2}\right]$ of mass $304.8 \mathrm{kDa}$ (Figure 3; component $\mathrm{D}$ ). There was no sign of a species corresponding to a fully formed 5-fold axis 5( $\left.\mathrm{CP}_{2}: \mathrm{TR}\right)$, suggesting that these species result from formation of a 3 -fold axis assembly intermediate. The $\left[5\left(\mathrm{CP}_{2}: \mathrm{TR}\right)+5 \mathrm{CP}_{2}\right]$ species has only recently been tentatively assigned and could correspond to a 5-fold intermediate with additional subunits 

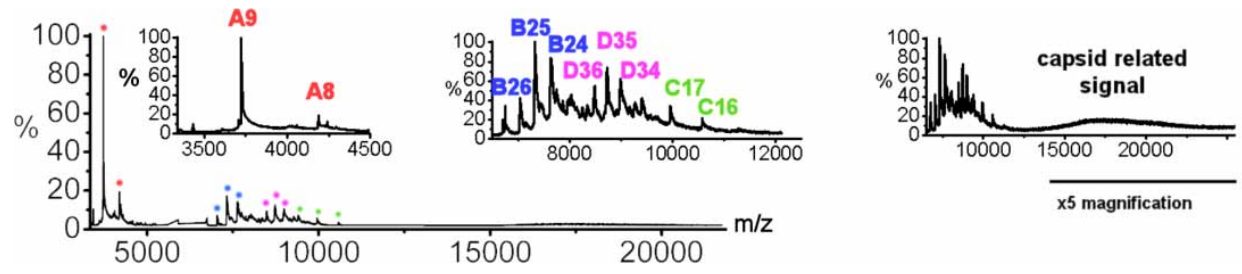

Figure 3. Mass spectrum of a TR-mediated assembly reaction. Virus reassembly reaction in the ratio 2:1 $\mathrm{CP}_{2}$ :TR after $30 \mathrm{~min}$. The spectra were acquired over the range $\mathrm{m} / \mathrm{z} 500-30,000$, for sample in ammonium acetate $(40 \mathrm{mM})$ at $\mathrm{pH} 5.2-5.7$. The observed intermediates are labelled as follows; $\mathrm{A}=\mathrm{CP}_{2}: \mathrm{TR}$; $\mathrm{B}=182.7 \mathrm{kDa}$, previously assigned as $\left[3\left(\mathrm{CP}_{2}: \mathrm{TR}\right)+3 \mathrm{CP}_{2}\right], \mathrm{C}=169.2 \mathrm{kDa}$ species assigned as $\left[3\left(\mathrm{CP}_{2}: \mathrm{TR}\right)+2 \mathrm{CP}_{2}+\mathrm{CP}\right]$, and $\mathrm{D}=304.8 \mathrm{kDa}$ species assigned for the first time as $\left[5\left(\mathrm{CP}_{2}: \mathrm{TR}\right)+5 \mathrm{CP}_{2}\right]$. Available in colour online.

bound. A minimal $5\left(\mathrm{CP}_{2}: \mathrm{TR}\right)$ possible intermediate could be unstable, explaining why it has not been seen before. Alternatively, combinatorial analysis (Stansifer and Twarock, pers. comm.) suggests that the 3 -fold species corresponds to the more probable of these two possibilities, implying that despite the suggestive 5-fold stoichiometry this intermediate is more likely to form as an extension of the 3 -fold stoichiometry, $\left[3\left(\mathrm{CP}_{2}: \mathrm{TR}\right)+3 \mathrm{CP}_{2}\right]$. Once such a structure forms the phage has ensured that the protein will assemble a $T=3$ structure and not a $T=1$ capsid. Additional experiments, including ones with isotopically-labelled protein, showed that all the intermediates were competent for further assembly, i.e. were on the assembly pathway and that the unit of capsid growth is a $\mathrm{CP}_{2}$.

The data described above suggest a detailed molecular mechanism for $T=3$ shell formation (Figure 4) in which TR binding to the CP dimer in solution induces a conformational change in the FG-loops of the protein monomers yielding an A/B-like dimer from a C/C-like precursor. In other words, the TR stem-loop acts as an allosteric ligand. Whilst this is a satisfactory explanation of the data for in vitro reassembly reactions with multiple copies of the TR: $\mathrm{CP}_{2}$ complex present it does not necessarily apply to assembly in vivo where the genomic RNA contains only one copy of the TR stem-loop. There are a number of options in this case. Capsid assembly might need only to be initiated via a single TR stem-loop induced conformer switch. Alternatively, RNA sequences outside the TR region may mimic TR action during the assembly process and this would be consistent with a recent cryo-electron microscopy (cryo-EM) reconstruction of the wild-type phage particle at intermediate resolution [33]. This shows an array of RNA directly beneath the protein shell but with the average density below an A/B dimer differing from that below the $\mathrm{C} / \mathrm{Cs}$ (Figure 5(A)).

In order to examine the putative role(s) of TR flanking sequences during assembly, we carried out reassembly reactions with RNA oligonucleotides carrying genomic $5^{\prime}$ or $3^{\prime}$ flanking sequences attached to the TR site (Figure 1, lower panel; [28]). All of the RNAs promote capsid formation, as judged by the positions of migration on gels of the RNAs at higher protein concentrations (Figure 5(B), upper panels). Perhaps, surprisingly the longer RNAs seem to be less efficient than TR in this reaction, cf. lanes 4-6 in the various gels. The two longest RNAs also produce smears that migrate slower than the capsid species (lanes 7-9). Electron micrographs suggest that this is the result of the formation of partially formed capsids. These results can be rationalized by reference to the model of the assembly reaction (Figure 4). In the presence of TR there are only two significantly populated types of protein conformer, A/B and $\mathrm{C} / \mathrm{C}$, and these can each be added to a growing $T=3$ capsid. For the longer RNAs, however, there is the possibility that more than one CP dimer could bind to a single RNA, resulting in a complex mixture of unknown conformations of proteins. As a consequence, the concentrations 


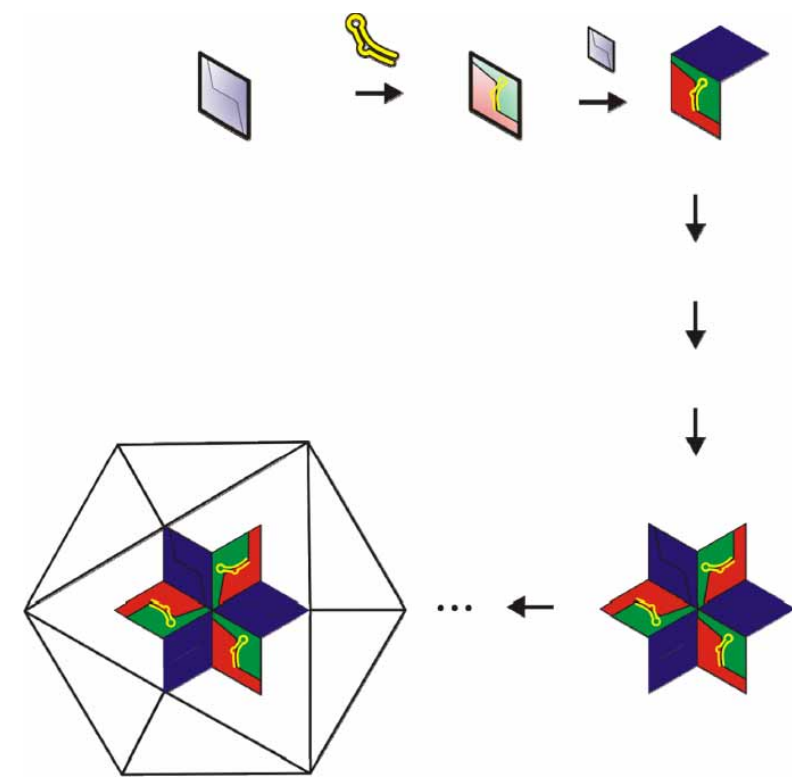

Figure 4. Model for the initiation of $T=3$ capsid assembly. A pair of chemically equivalent (C/C-like) subunits in solution (top left, shaded pink) binds a TR RNA resulting in a conformational switch into an A/B-like asymmetric dimer (shaded green and blue). This complex binds an additional C/C-like dimer creating the first higher order intermediate on the pathway to capsid formation. A variety of sub-pathways involving addition of CP dimers (arrows) can then generate the major assigned intermediate encompassing the first 3-fold axis of the virus particle. Adapted from Ref. [29]. Available in colour online.

of species able to participate in capsid formation are potentially lowered. It appears that the longer RNAs are acting as 'traps' on the assembly pathway.

In vivo of course the assembly reaction has to package a much longer genomic RNA and ensure that it acquires the complex 3D-fold seen in cryo-EM reconstructions that includes a second inner shell of RNA as well as the network seen directly below the CP (Figure 5(A)). The trapping of proteins seen in the reassembly reaction with the shorter fragments cannot simply scale up with the length of the RNA or assembly in vivo would never take place. A number of proposals have been made about the assembly of positive-strand RNA viruses, including the idea that only nascent transcripts from the polymerase are able to be packaged. There is some evidence of such requirements in pariacoto virus [37], a member of the Nodaviridae and brome mosaic virus (BMV) [1]. It has been argued that this type of packaging mechanism is driven by the fact that nascent transcripts fold using short range base pairing to create an RNA conformation easily adapted to the small volumes of the final capsid [15]. The apparent 'trapping' effect of the TR extension RNAs could be the result of such a mechanism. In order to test this idea, we have cloned an internal MS2 genomic fragment $928 \mathrm{nt}$ long that encompasses the TR stem-loop signal. This has also been used successfully in in vitro reassembly experiments (Figure 5(B), lower panel). Once again a smear migrating more slowly than the bona fide $T=3$ capsid is created and electron micrographs suggest that this may be due to aggregation of partially formed capsids. Clearly there is no absolute requirement to have nascent transcripts, even with a large sub-genomic fragment, but at high protein:RNA molar ratios, RNAs may end up being shared between assembling capsids creating aggregates. Since there is little time during a phage infection to resolve such structures, the ratio of RNA:CP must be tightly controlled in vivo to avoid this problem. 

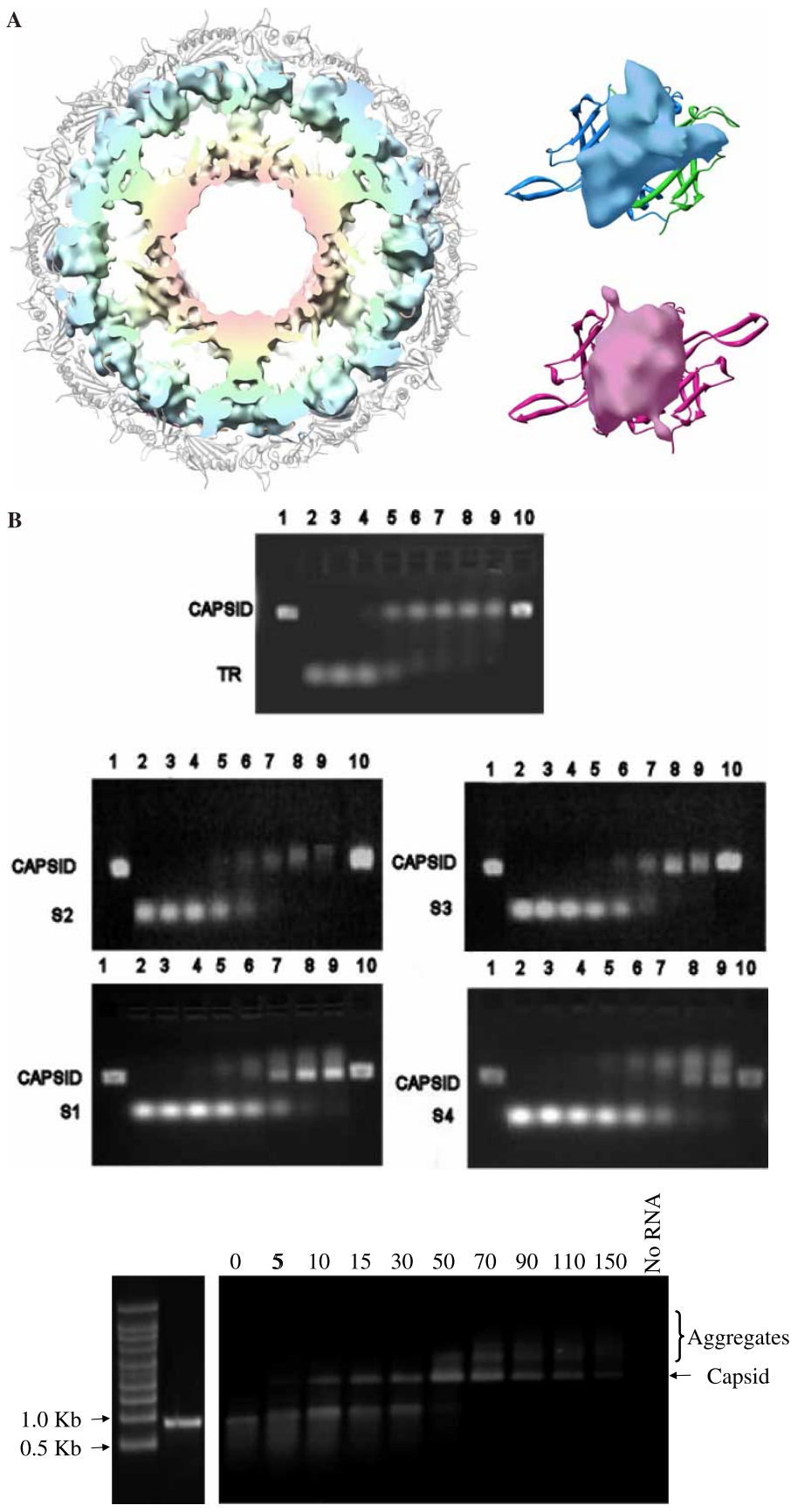

Figure 5. In vitro assembly of capsids using RNA fragments. (A) Cartoon (left) showing a cross-section view of the cryo-EM reconstruction of the MS2 wild-type phage virion [33]. Regions of the cryo-EM density corresponding to the MS2 CP shell (shown in cartoon representation in pale grey) have been masked away, leaving density for encapsidated genomic RNA. Genomic RNA is observed in two shells. The first lies directly beneath the protein capsid and maps precisely to the known binding sites for RNA on the icosahedral CP lattice. The second is at lower radius, and the two shells are linked by density on each icosahedral 5-fold axis. On the right the averaged electron density beneath $\mathrm{A} / \mathrm{B}$ (blue/green) and $\mathrm{C} / \mathrm{C}$ (red/red) dimers in this structure 
These data suggest that RNA mimics that encompass viral CP binding sites could be effective anti-viral agents because they would either trap CPs in unproductive aggregates or redirect the normal assembly pathway. Viral RNA and its protective CPs have to make very many precise interactions with each other, and if such contacts occur improperly anywhere during the process the formation of infectious progeny virus should be impaired. Current therapeutics do not target viral self-assembly pathways but reagents with such potential could prove to be potent inhibitors of viral assembly pathways.

\section{Materials and methods \\ Proteins, RNAs and reagents}

Wild-type bacteriophage MS2, maintained in $10 \mathrm{mM}$ Tris $-\mathrm{HCl}$ (pH 7.5), $100 \mathrm{mM} \mathrm{NaCl}, 0.1 \mathrm{mM}$ $\mathrm{MgCl}_{2}$ and $0.01 \mathrm{mM}$ EDTA (buffer A), was a gift from Professor David S. Peabody, University of New Mexico (Albuquerque). Wild-type, recombinant MS2 CP was over-expressed in E. coli and purified using previously described methods $[18,30]$. Purified $T=3$ capsids were stored in $10 \mathrm{mM}$ HEPES ( $\mathrm{pH}$ 7.2), $100 \mathrm{mM} \mathrm{NaCl}$ and $1 \mathrm{mM}$ EDTA. $\mathrm{CP}_{2}$ for reassembly reactions was obtained by treating the recombinant capsids with glacial acetic acid to induce capsid dissociation to disassembled CP monomers, followed by exchange into $20 \mathrm{mM}$ acetic acid to promote dimerization [31]. To ensure that complete disassembly had occurred before reassembly, the $\mathrm{CP}_{2}$ were examined using negative stain EM [30]. Synthetic oligonucleotides were prepared as described previously [19].

The $928 \mathrm{nt}$ sub-genomic fragment was prepared by in vitro transcription from a cDNA template corresponding to nucleotides 1419-2346 of the MS2 genome using a MEGAscript T7 kit (Ambion) according to the manufacturer's instructions. The cDNA template was generated by PCR using the forward primer GATAATACGACTCACTATAGGGGTCGCTGAATGGATCAGC and reverse primer CTGTAAACACTCCGTTCCCTACA from the plasmid pSmart_3528. The plasmid pSmart-3528 is a pSMART (Lucigen Corporation, Middleton, WI, USA) plasmid encompassing cDNA corresponding to nucleotides 19-3550 of the MS2 genomic sequence (AN: V00642) as verified by automated DNA sequencing.

\section{MS2 capsid reassembly reactions}

Reassembly reactions were performed with the short oligonucleotides ( $\mathrm{S} 1-\mathrm{S} 4$ and TR) by titrating the RNA with increasing amounts of $\mathrm{CP}_{2}$ in reassembly buffer $\mathrm{A}(20 \mathrm{mM}$ Tris-acetate, $\mathrm{pH} 7.0-7.5)$, allowing the reactions to proceed for $1 \mathrm{~h}$ on ice and then analysing the products on agarose-acrylamide mixed gels (Figure 5). The recombinant $T=3$ capsid was used as a marker (lanes 1 and 10). The RNAs ( $1 \mu \mathrm{M}$; lanes 2) were titrated with $0.1,0.2,0.5,1,2,4$ and $5 \mu \mathrm{M} \mathrm{CP}_{2}$ (lanes 3-9).

Equivalent experiments with the large RNA fragment were carried out by titrating the RNA with increasing amounts of $\mathrm{CP}_{2}$ in reassembly buffer $\mathrm{B}\left(40 \mathrm{mM} \mathrm{NH}_{4} \mathrm{OAc}, 1 \mathrm{mM} \mathrm{Mg}(\mathrm{OAc})_{2}\right.$, $\mathrm{pH}=5.8-6.5)$. The reassembly reactions were performed at 50 nM RNA with 5-150 molar excess of $\mathrm{CP}_{2}$ in a $20 \mu \mathrm{l}$ final reaction volume and allowed to proceed for $3 \mathrm{~h}$ at $4{ }^{\circ} \mathrm{C} .5 \mu \mathrm{L}$ of loading buffer ( $40 \%$ sucrose, $0.25 \%$ bromophenol blue) was then added to each reaction prior to

are shown. Adapted from Ref. [33]. (B) Upper panel: agarose-acrylamide gels of reassembly reactions with short fragments. Samples were - lanes 1 and $10=$ recombinant $T=3$ shells as a control; lanes $2-$ $9=$ RNAs (TR, S1-S4, as indicated) at $1 \mu \mathrm{M}$ with $0,0.1,0.2,0.5,1,2,4$ and $5 \mu \mathrm{M} \mathrm{CP}_{2}$ added. Lower panel: shows an agarose gel of a reassembly reaction with the $928 \mathrm{nt}$ fragment (Figure 1) at differing molar excesses of CP dimers (indicated above each lane). Available in colour online. 
agarose electrophoreses in a $0.8 \%$ agarose gel at $80 \mathrm{~V}$ for $60 \mathrm{~min}$. Agarose gels were stained post electrophoreses in TBE running buffer containing $5 \mu \mathrm{g} / \mathrm{ml} \mathrm{EtBr}$ and visualized at $254 \mathrm{~nm}$.

\section{Acknowledgements}

We thank The Wellcome Trust, The Leverhulme Trust and the UK BBSRC for financial support, Professor David Peabody, University of New Mexico for his gift of wild-type MS2 bacteriophage, and Dr Abdul Rashid for oligonucleotide synthesis. OR, KT and SF thank The Wellcome Trust, the University of Leeds Interdisciplinary Institute in Bionanosciences, and the EU Marie Curie scheme for PhD studentship support, respectively. NAR holds a University of Leeds Research Fellowship.

\section{Note}

1. Present address: BMRC, Sheffield Hallam University, Howard Street S1 1WB, Sheffield, UK.

\section{References}

[1] P. Annamalai and A.L.N. Rao, Packaging of brome mosaic virus subgenomic RNA is functionally coupled to replication-dependent transcription and translation of coat protein, J. Virol. 80(20) (2006), pp. 10096-10108.

[2] D. Beckett and O.C. Uhlenbeck, Ribonucleoprotein complexes of R17 coat protein and a translational operator analog, J. Mol. Biol. 204 (1988), pp. 927-938.

[3] D. Beckett, H.N. Wu, and O.C. Uhlenbeck, Roles of operator and non-operator RNA sequences in bacteriophage R17 capsid assembly, J. Mol. Biol. 204(4) (1988), pp. 939-947.

[4] J. Carey and O.C. Uhlenbeck, Kinetic and thermodynamic characterization of the R17-coat protein ribonucleic-acid interaction, Biochemistry 22(11) (1983), pp. 2610-2615.

[5] J. Carey, V. Cameron, P.L. Dehaseth, and O.C. Uhlenbeck, Sequence-specific interaction of R17-coat protein with its ribonucleic-acid binding-site, Biochemistry 22(11) (1983), pp. 2601-2610.

[6] D. Caspar and A. Klug, Physical principles in the construction of regular viruses, Cold Spring Harb. Symp. Quant. Biol. 27 (1962), pp. 1-24.

[7] M.A. Convery, S. Rowsell, N.J. Stonehouse, A.D. Ellington, I. Hirao, J.B. Murray, D.S. Peabody, S.E.V. Phillips, and P.G. Stockley, Crystal structure of an RNA aptamer protein complex at 2.8 angstrom resolution, Nat. Struct. Biol. 5(2) (1998), pp. 133-139.

[8] F.H.C. Crick and J.D. Watson, Structure of small viruses, Nature 177(4506) (1956), pp. 473-475.

[9] R. Golmohammadi, K. Valegård, K. Fridborg, and L. Liljas, The refined structure of bacteriophage MS2 at 2.8 A resolution, J. Mol. Biol. 234 (1993), pp. 620-639.

[10] E. Grahn, N.J. Stonehouse, J.B. Murray, S. Van den Worm, K. Valegard, K. Fridborg, P.G. Stockley, and L. Liljas, Crystallographic studies of RNA hairpins in complexes with recombinant MS2 capsids: Implications for binding requirements, RNA-A Publ. RNA Soc. 5(1) (1999), pp. 131-138.

[11] H.R. Hill, N.J. Stonehouse, S.A. Fonseca, and P.G. Stockley, Analysis of phage MS2 coat protein mutants expressed from a reconstituted phagemid reveals that proline 78 is essential for viral infectivity, J. Mol. Biol. 266(1) (1997), pp. 1-7.

[12] W.T. Horn, K. Tars, E. Grahn, C. Heigstrand, A.J. Baron, H. Lago, C.J. Adams, D.S. Peabody, S.E.V. Phillips, N.J. Stonehouse et al., Structural basis of RNA binding discrimination between bacteriophages $Q$ beta and MS2, Structure 14(3) (2006), pp. 487-495.

[13] W.M. Jou, G. Haegeman, M. Ysebaert, and W. Fiers, Nucleotide sequence of the gene coding for the bacteriophage MS2 coat protein, Nature 237 (1972), pp. 82-88.

[14] H. Lago, A.M. Parrott, T. Moss, N.J. Stonehouse, and P.G. Stockley, Probing the kinetics of formation of the bacteriophage MS2 translational operator complex: Identification of a protein conformer unable to bind RNA, J. Mol. Biol. 305(5) (2001), pp. 1131-1144.

[15] S.B. Larson and A. McPherson, Satellite tobacco mosaic virus RNA: Structure and implications for assembly, Curr. Opin. Struct. Biol. 11 (2001), pp. 59-65.

[16] C.M. Ling, P.P. Hung, and L.R. Overby, Independent assembly of $Q \beta$ and MS2 phages in doubly infected E. coli, Virology 40 (1970), pp. 920-929.

[17] H.F. Lodish, Regulation of in vitro protein synthesis by bacteriophage RNA by RNA tertiary structure, RNA Phages (1975), p. 301. 
[18] R.A. Mastico, S.J. Talbot, and P.G. Stockley, Multiple presentation of foreign peptides on the surface of an RNA-free spherical bacteriophage capsid, J. Gen. Virol. 74 (1993), pp. 541-548.

[19] J.B. Murray, A.K. Collier, and J.R. Arnold, A general purification procedure for chemically synthesized oligoribonucleotides, Anal. Biochem. 218(1) (1994), pp. 177-184.

[20] C.Z. Ni, R. Syed, R. Kodandapani, J. Wickersham, D.S. Peabody, and K.R. Ely, Crystal-structure of the MS2 coat protein dimer - implications for RNA-binding and virus assembly, Structure 3(3) (1995), pp. 255-263.

[21] W. Paranchych, Attachment, ejection and penetration stages of the RNA phage infectious process, RNA phages (1975), pp. 85-112.

[22] M.G. Rossmann and J.E. Johnson, Icosahedral RNA virus structure, Ann. Rev. Biochem. 58 (1989), pp. 533-573.

[23] M.G. Rossmann, C. Abadzapatero, J.W. Erickson, and H.S. Savithri, RNA-protein interactions in some small plant-viruses, J. Biomol. Struct. Dyn. 1(2) (1983), pp. 565-579.

[24] S. Rowsell, N.J. Stonehouse, M.A. Convery, C.J. Adams, A.D. Ellington, I. Hirao, D.S. Peabody, P.G. Stockley, and S.E.V. Phillips, Crystal structures of a series of RNA aptamers complexed to the same protein target, Nat. Struct. Biol. 5(11) (1998), pp. 970-975.

[25] A. Schneemann, The structural and functional role of RNA in icosahedral virus assembly, Ann. Rev. Microbiol. 60 (2006), pp. 51-67.

[26] P.K. Sorger, P.G. Stockley, and S.C. Harrison, Structure and assembly of turnip crinkle virus II: Mechanism of reassembly in vitro, J. Mol. Biol. 191(4) (1986), pp. 639-658.

[27] P.G. Stockley, N.J. Stonehouse, and K. Valegard, Molecular mechanism of Rna phage morphogenesis, Int. J. Biochem. 26(10-11) (1994), pp. 1249-1260.

[28] P.G. Stockley, A.E. Ashcroft, S. Francese, G.S. Thompson, N.A. Ranson, A.M. Smith, S.W. Homans, and N.J. Stonehouse, Dissecting the fine details of assembly of a $\mathrm{T}=3$ phage capsid, Comput. Math. Methods Med. 6 (2005), pp. 119-125.

[29] P.G. Stockley, O. Rolfsson, G.S. Thompson, G. Basnak, S. Francese, N.J. Stonehouse, S.W. Homans, and A.E. Ashcroft, A simple, RNA-mediated allosteric switch controls the pathway to formation of a $\mathrm{T}=3$ viral capsid, J. Mol. Biol. 369(2) (2007), pp. 541-552.

[30] N.J. Stonehouse and P.G. Stockley, Effects of amino acid substitution on the thermal stability of MS2 capsids lacking genomic RNA, FEBS Lett. 334(3) (1993), pp. 355-389.

[31] T. Sugiyama and D. Nakada, Control of translation of MS2 RNA cistrons by MS2 coat protein, Biochemistry 57 (1967), pp. 1744-1750.

[32] K. Tars, M. Bundule, K. Fridborg, and L. Liljas, The crystal structure of bacteriophage GA and a comparison of bacteriophages belonging to the major groups of E. coli leviviruses, J. Mol. Biol. 271(5) (1997), pp. 759-773.

[33] K. Toropova, G. Basnak, R. Twarock, P.G. Stockley, and N.A. Ranson, The three-dimensional structure of genomic RNA in bacteriophage MS2: Implications for assembly, J. Mol. Biol. 375(3) (2008), pp. 824-836.

[34] K. Valegard, L. Liljas, K. Fridborg, and T. Unge, The three-dimensional structure of the bacterial virus MS2, Nature 345 (1990), pp. 36-41.

[35] K. Valegard, J.B. Murray, P.G. Stockley, N.J. Stonehouse, and L. Liljas, Crystal structure of a bacteriophage-RNA coat protein-operator complex, Nature 371(6498) (1994), pp. 623-626.

[36] K. Valegard, J.B. Murray, N.J. Stonehouse, S. vandenWorm, P.G. Stockley, and L. Liljas, The threedimensional structures of two complexes between recombinant MS2 capsids and RNA operator fragments reveal sequence-specific protein-RNA interactions, J. Mol. Biol. 270(5) (1997), pp. $724-738$.

[37] P.A. Venter and A. Schneemann, Assembly of two independent populations of flock house virus particles with distinct RNA packaging characteristics in the same cell, J. Virol. 81(2) (2007), pp. 613-619.

[38] N. Wei and T.J. Morris, Interactions between viral coat protein and a specific binding region on turnip crinkle virus-RNA, J. Mol. Biol. 222(3) (1991), pp. 437-443.

[39] G.W. Witherell, J.M. Gott, and O.C. Uhlenbeck, Specific interaction between RNA phage coat proteins and RNA, Prog. Nucleic Acid Res. Mol. Biol. 40 (1991), pp. 185-220.

[40] K. Wong and W. Paranchych, Effect of ribonuclease on penetration of R17 phage A-protein and RNA, Can. J. Microbiol. 22(6) (1976), pp. 826-831.

[41] A. Zlotnick, R. Aldrich, J.M. Johnson, P. Ceres, and M.J. Young, Mechanism of capsid assembly for an icosahedral plant virus, Virology 277 (2000), pp. 450-456. 


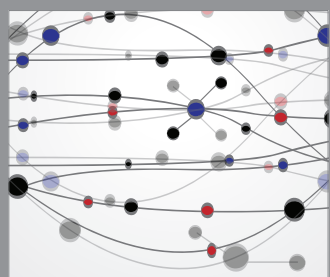

The Scientific World Journal
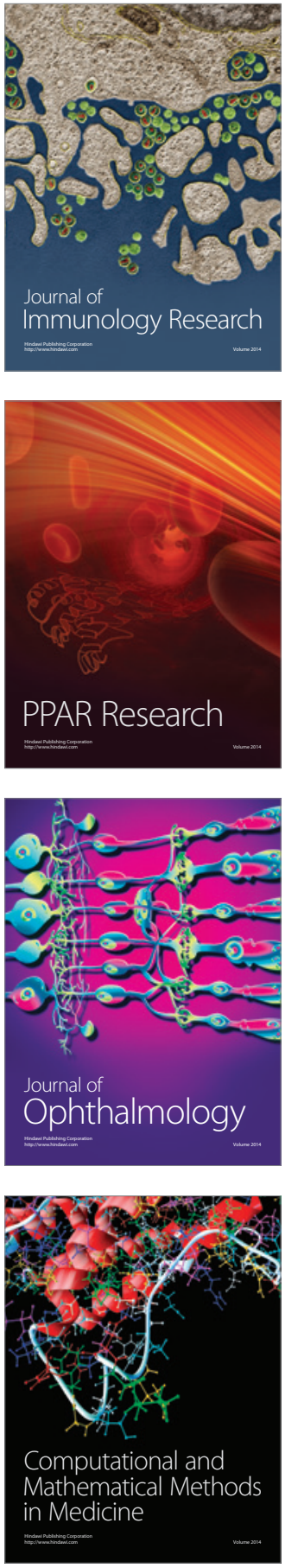

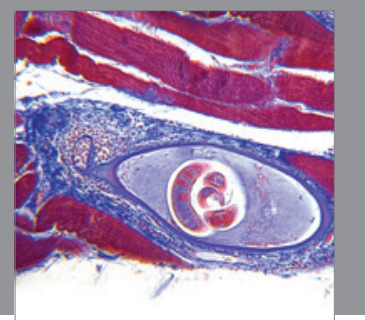

Gastroenterology

Research and Practice
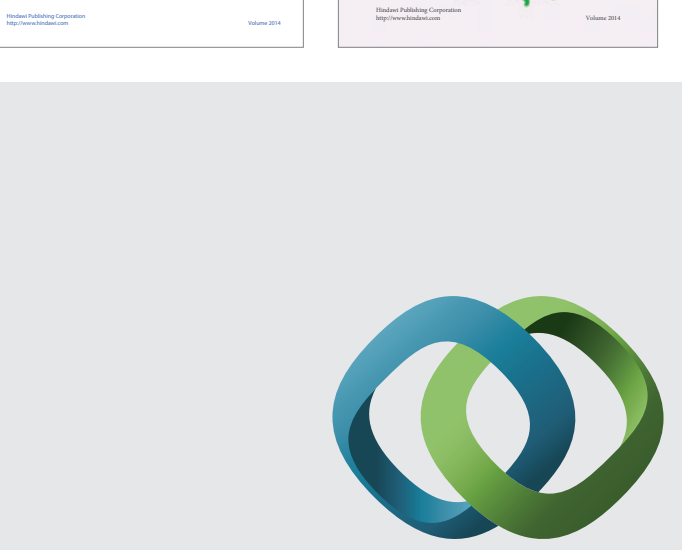

\section{Hindawi}

Submit your manuscripts at

http://www.hindawi.com
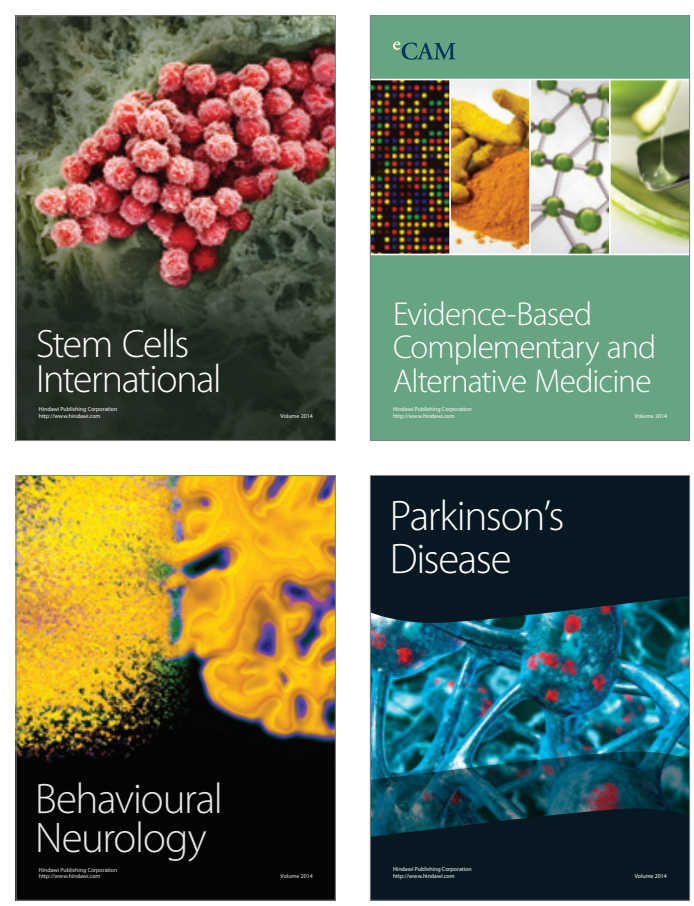

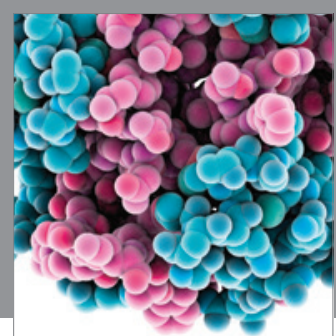

Journal of
Diabetes Research

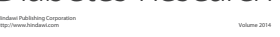

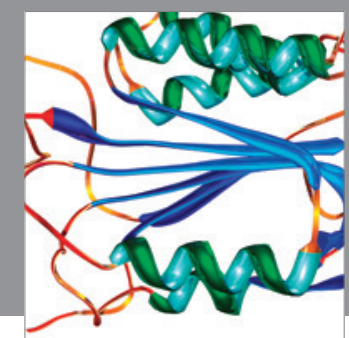

Disease Markers
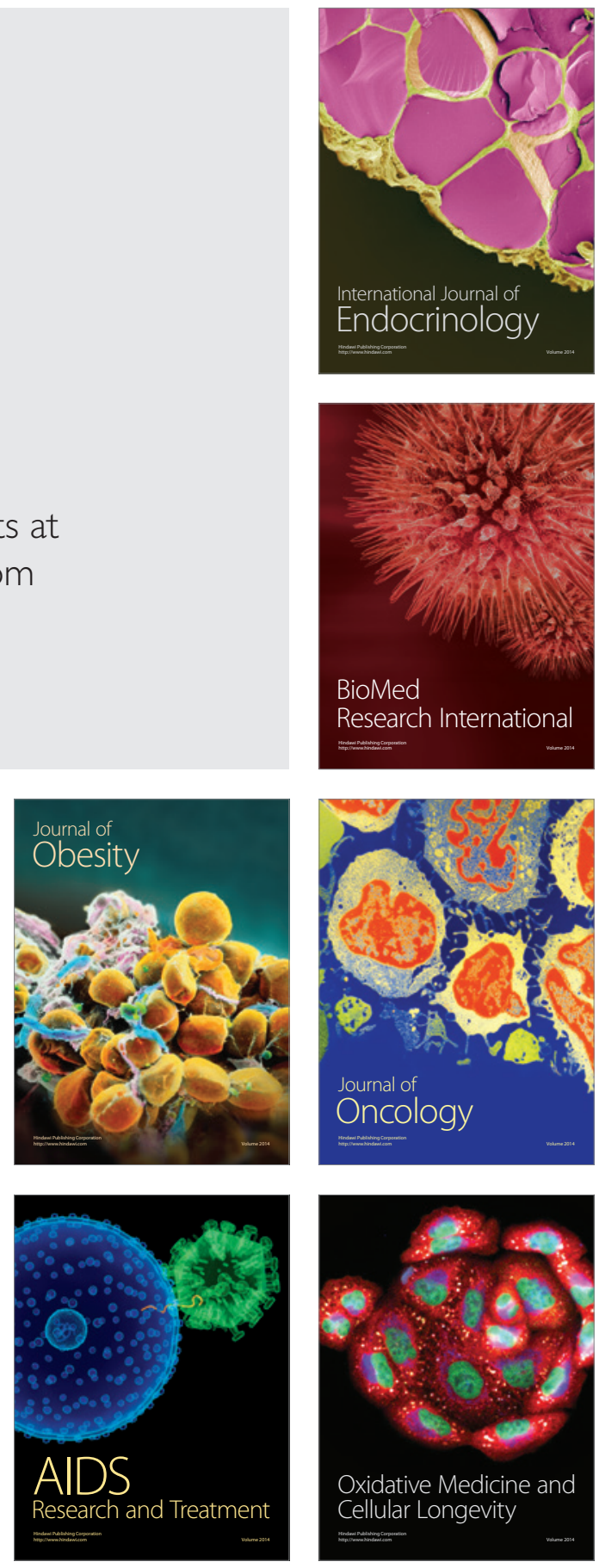